\title{
The Role of Dynamic MRI with Diffusion Weighted Images in Evaluation of Portal Vein Thrombosis in Hepatic Patients \\ ${ }^{1}$ Suzan Bahig Ali, ${ }^{1}$ Asmaa Magdy Salama, ${ }^{2}$ Mostafa Farag Elshafie, ${ }^{*}$ Martina Fares Fayez Shogry \\ Department of Radiology, Faculty of Medicine, ${ }^{1}$ Ain Shams University \\ Department of Radiology, ${ }^{2}$ Theodore Bilharz Research Institute \\ *Corresponding author: Martina F.F Shogry, Mobile: 01285352072, Email: martinafares9@ gmail.com
}

\begin{abstract}
Background: hepatocellular carcinoma (HCC) is the fifth most common tumor worldwide and its incidence is increasing especially in western nations. HCC may be associated with portal vein thrombosis which could be either benign or malignant. Aim of the Work: to detect the role of diffusion MR imaging in the differentiation between benign and malignant portal vein thrombosis in patients with HCC. Materials and Methods: prospective study carried on 30 patients with liver cirrhosis with pathological or radiological proof of HCC, associated with visible portal vein thrombosis. Dynamic MRI examination and diffusion weighted imaging were performed for all patients. ADC values and ratios were calculated for both HCC and thrombus. Definite accepted radiological criteria were used as a standard of reference to detect the nature of the thrombus and discriminate between bland ones and neoplastic ones. Results: we found that the ratio between the ADC of the thrombus and ADC of the HCC was significantly different between the malignant and bland cohorts and a cutoff value of 1.25 for the ADC ratio helped in differentiation between malignant and benign portal vein thrombi with $85 \%$ sensitivity and $81 \%$ specificity. There was also obvious statistical significant difference between the ADC values of the bland and neoplastic thrombi. Conclusion: diffusion weighted MR imaging has a great role in differentiation between the benign and malignant portal vein thrombosis by measuring the ADC ratio between the thrombus and HCC.
\end{abstract}

Keywords: Portal vein thrombosis, Liver cirrhosis, HCC, Diffusion weighted imaging, ADC value, ADC ratio.

\section{INTRODUCTION}

Hepatocellular carcinoma (HCC) is the fifth most common tumor worldwide and its incidence is increasing especially in western nations. HCC may be associated with portal vein thrombosis which could be either benign or malignant ${ }^{(\mathbf{1}}$. Neoplastic portal vein thrombi in patients with HCC gravely affect prognosis and subsequent treatment options. These patients are considered unsuitable for most therapeutic options, including thermal or chemical ablation, trans-arterial chemoembolization, liver resection, and even orthotopic liver transplantation. Five year survival after surgical resection is $12 \%-$ $39 \%$ in patients with neoplastic vascular invasion and $59 \%$ in those without. Such patients usually undergo palliative or experimental treatment ${ }^{(1,2)}$. Although the reference standard in the diagnosis of the malignant portal vein thrombosis is the pathologic examination, in clinical practice, diagnostic imaging plays a pivotal role ${ }^{(3)}$. Biopsy of portal vein has many drawbacks; it relies on the skills of the radiologist and the size of the affected vein and if performed improperly, the tumor cells could be obtained from adjacent HCC rather than from the thrombus, leading to misdiagnosis, or the amount of the biopsy material could be insufficient for evaluation. Moreover, portal vein thrombus biopsy is an invasive procedure with associated risks of bleeding especially in these patients with coexistent coagulation defects. On the other hand, demonstration of arterial flow within the thrombus by using spectral Doppler US is $100 \%$ specific for tumor thrombus. Also, contrast-enhanced US has been demonstrated to be $88 \%$ sensitive and $100 \%$ specific in the diagnosis of malignant portal vein thrombosis ${ }^{(3)}$. These figures are similar to those obtained at contrast-enhanced CT, with a sensitivity of $86 \%$ and a specificity of $100 \%{ }^{(4)}$. Therefore, in practice, diagnosis is often done with a combination of laboratory and imaging findings. MRI have a role in differentiating malignant from bland thrombosis, malignant thrombus is characterized at imaging by expansile dilatation of the portal vein and intermediate to high signal intensity on $\mathrm{T} 2$ images in comparison to the normal portal vein caliber and low T2 signal seen in bland thrombosis "attributed to hemosiderin within the thrombus". A malignant thrombus also shows arterial neovascularity with enhancement similar to the primary tumor and often is contiguous with the primary tumor ${ }^{(5)}$. Diffusion imaging is an MR technology that helps more in tissue characterization. In malignant tissue, the diffusion of water molecule is more restricted due to higher lesion cellularity, so lower ADC values are noted as compared to benign lesions. Also, DW imaging does not require contrast medium 
administration; therefore it can be safely done in patients with contraindications to contrast media ${ }^{(2)}$.

\section{AIM OF THE WORK}

This study aims to determine the role of diffusion MR imaging in the differentiation between benign and malignant portal vein thrombosis in patients with HCC.

\section{PATIENTS AND METHODS}

Patients: The study was prospectively carried on 30 patients ( 28 males and 2 females) between October 2017 and May 2018. The mean age of the patients was 60.3. All patients presented with liver cirrhosis with pathological or radiological proof of HCC and visible portal vein thrombosis. Patients with previous hepatic intervention whether surgical, loco regional or systemic therapy were excluded considering that therapeutic options may affect the original tumor's cellularity and pattern and therefore its diffusion characteristics. Also, patients with malignancy other than HCC were excluded. MR examination of the liver was performed with a 1.5 Tesla machine (Achieva, Philips medical system, Eindhoven, Netherlands) with the use of a dedicated abdominal eight-channel surface coil. Ethical approval: The study was approved by the Ethics Board of Ain Shams University and an informed written consent was taken from each participant in the study. Technique: Precontrast images of the liver using the sequences (Axial T1 TFE, Axial T2 TSE, and Axial T2 SPAIR), The acquisition parameters for T1WI were TR $10 \mathrm{msec}$, TE $4.6 \mathrm{msec}$, field of view $300-350 \mathrm{~mm}$, slice thickness $7 \mathrm{~mm}$, flip angle 15 and for T2WI were TR $1000 \mathrm{msec}$, TE $80 \mathrm{msec}$, field of view 300-350 $\mathrm{mm}$, slice thickness $7 \mathrm{~mm}$, flip angle 90. Then Diffusion MR imaging was performed before the dynamic study using respiratory triggered fatsuppressed single-shot spin echo echoplanar sequence that combined the two diffusion (motionprobing) gradients before and after the $180^{\circ}$ pulse. The acquisition parameters were: TR $1700 \mathrm{msec}$, TE 76 msec, matrix $120 \times 95$ with a field of view as small as possible, slice thickness $10 \mathrm{~mm}$, slice gap 1-2 mm, scan time 3-4 min. We used b values of 0,500 and $1000 \mathrm{~s} / \mathrm{mm} 2$.Dynamic study was then performed after manual bolus injection of 0.1 $\mathrm{mmol} / \mathrm{kg}$ body weight of Gd-DTPA. Dynamic imaging using 3D fat-suppressed T1-weighted gradient echo sequence (THRIVE i.e. T1 high resolution isotropic volume examination). A dynamic series consisted of one pre contrast series followed by four successive post contrast series including early arterial, late arterial, and portal phases with 19-21 seconds intervals (17seconds for image acquisition with breath-holding and 2-4 seconds for re-breathing) this is followed by 5 -min delayed phase series. All patients were imaged at end expiration to limit the risk of image misregistration. Acquisition parameters were TR $4.4 \mathrm{msec}$., TE $2.1 \mathrm{msec}$., flip angle $10^{\circ}$, matrix size, $172 \times 163$, field of view $300-350 \mathrm{~mm}$ and slice thickness $2 \mathrm{~mm}$. Imaging analysis and interpretation: Images were analyzed using the available workstation (Phillips Extended MR Workspace, 2.6.3.5 Netherlands) by experienced abdominal radiologists. The morphological features of the lesion and the portal vein thrombosis were studied including the size of HCC, diameter of the portal vein and the distance between the lesion and the thrombosed portal vein. Assessment of the enhancement pattern of the lesion and the thrombus was done. Oval regions of interest (ROIs) were drawn manually which included at least two-thirds of the area of the HCC and at least two-thirds of the area of the portal vein thrombus, directly on the corresponding ADC image to obtain the ADC values. We enlarged the images and placed the ROI within the thrombus to minimize volume averaging. We tried to avoid including any area outside the tumor and the thrombus in the ROI. The mean and standard deviation of the ADC of hepatic tumor, the neoplastic thrombi, and the bland thrombi were calculated. Also, we calculate the ratio of the $\mathrm{ADC}$ of the thrombus to the $\mathrm{ADC}$ of the HCC (ADC ratio) in both benign and malignant cohorts. Statistical analysis: The ADC values of the HCC and thrombus as well as the ADC ratio were compared in each group by using the t-test. Analysis was performed and a $\mathrm{P}$ value of 0.05 was chosen as the threshold for statistical significance.

\section{RESULTS}

Among the studied 30 patients, 16 patients diagnosed as malignant portal vein thrombosis and 14 patients diagnosed as bland portal vein thrombosis according to the standard reference criteria as shown in figure 1 and 2. 


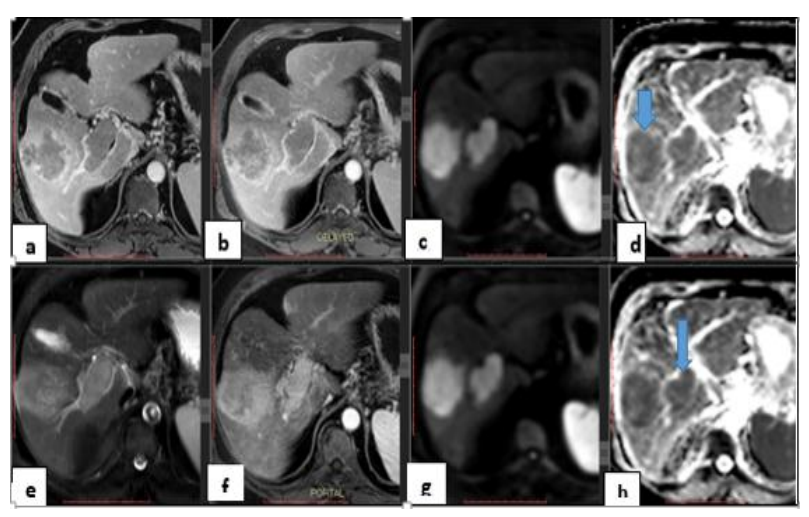

Figure 1: Liver cirrhosis in 56 years old male patient. (a) Arterial phase, (b) Delayed phase, (c) Diffusion images B800, (d) ADC map showing enhancement pattern keeping with $\mathrm{HCC}$ with restricted diffusion and ADC value 817., (e) T2WI, (f) Portal phase (g) Diffusion images and (h) ADC map showing right portal vein thrombosis with $\mathrm{ADC}$ value 1007. ADC ratio = 1.233 keeping with malignant thrombus.

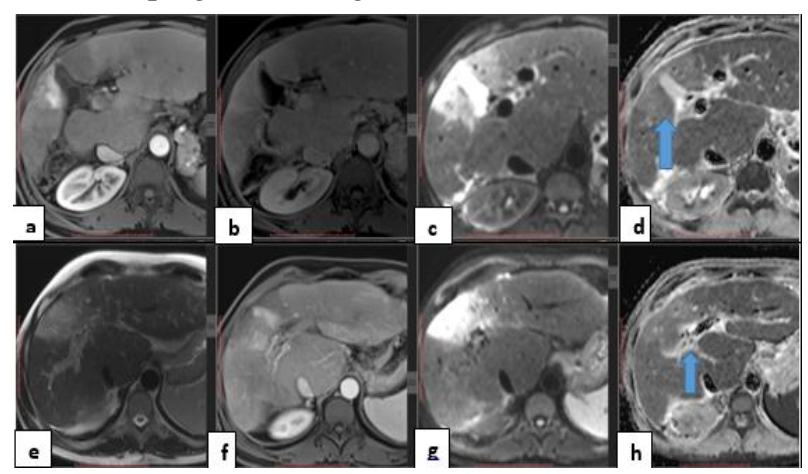

Figure 2: Liver cirrhosis in 53 years old male patient.(a) Arterial phase, (b) Delayed phase,(c) Diffusion images B800, (d)ADC map showing enhancement pattern keeping with $\mathrm{HCC}$ with restricted diffusion and ADC value 1058., (e) T2WI, (f) Portal phase (g) Diffusion images and (h) ADC map showing right portal vein thrombosis with $\mathrm{ADC}$ value 2133. $\mathrm{ADC}$ ratio $=2.016$ keeping with bland thrombus.

The size of the HCC in the studied patients with malignant portal vein thrombosis ranged from $3 \mathrm{~cm}$ to $13 \mathrm{~cm}$ with a mean of $6.86 \mathrm{~cm}$ and ranged from $1.5 \mathrm{~cm}$ to $7 \mathrm{~cm}$ with a mean of $2.46 \mathrm{~cm}$ in patients with benign portal vein thrombosis. The mean $\mathrm{ADC} \pm$ standard deviation of $\mathrm{HCC}$ in the neoplastic group was $856.25 \mathrm{~mm} 2 / \mathrm{sec} \pm 190.093$ and in the bland group were $888.71 \mathrm{~mm} 2 / \mathrm{sec} \pm$ 180.634. The mean ADC \pm standard deviation of malignant thrombus was $1051.25 \mathrm{~mm} 2 / \mathrm{sec} \pm$ 256.560 and in the bland thrombus were 1794.29 $\mathrm{mm} 2 / \mathrm{sec} \pm 463.828$. The mean $\mathrm{ADC}$ ratios \pm standard deviation of malignant thrombus was 1.27 \pm 0.4352 and in the bland thrombus were $2.09 \pm$
0.6667. The ADC values of the HCC and thrombus as well as the ADC ratio of the thrombus to the HCC were compared in each group by using the ttest. Analysis and a $\mathrm{P}$ value of 0.05 was chosen as the threshold for statistical significance. A cutoff value of 1.25 for the ADC ratio helped in discrimination between neoplastic and bland portal vein thrombi with $85 \%$ sensitivity and $81 \%$ specificity as shown in figures 3 and 4 .

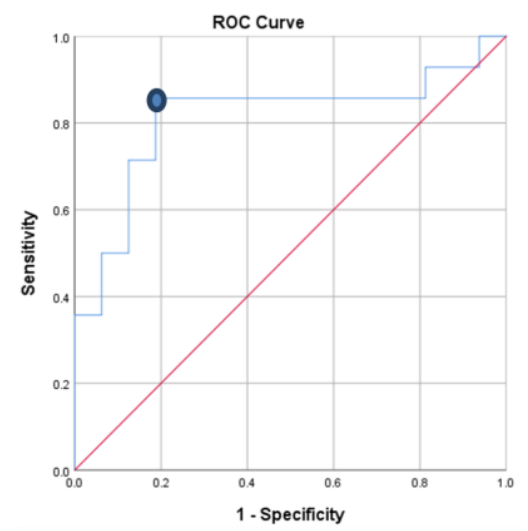

Figure 3: ADC ratio ROC curve. Blue dot refers to cutoff value at which maximum sensitivity and specificity.

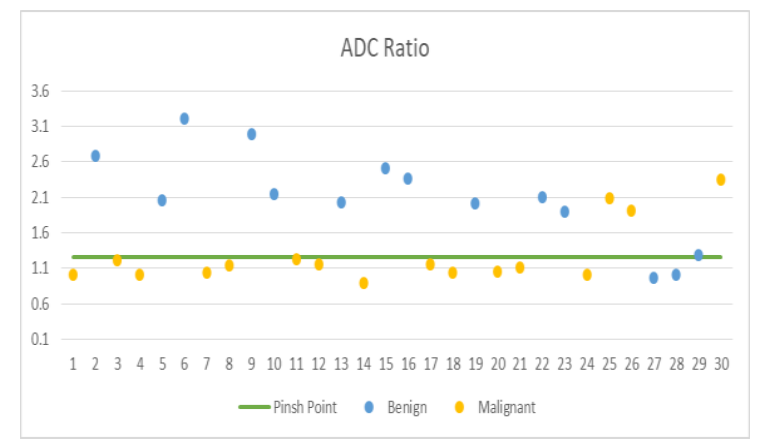

Figure 4: Malignant Versus Benign ADC ratios.

Standard of reference: It has been found that in cases of portal vein thrombosis in HCC patients, expansion of the involved vessel and enhancement of the thrombus in a pattern similar to that of the tumor are able to discriminate between the malignant and bland portal vein thrombus ${ }^{(6)}$. However, expansion of the portal vein is nonspecific and can also be observed in patients with portal hypertension without vein thrombosis (7). Moreover, some patients may have some contraindications for contrast material administration, due to impaired kidney function and/or previous history of contrast media allergic reaction ${ }^{(8)}$. According to the criteria used by Sandrasegaran et al. ${ }^{(9)}$, and supported by Sakata et $\boldsymbol{a l}{ }^{(\mathbf{1 0})}$; we considered the portal vein thrombosis as a malignant thrombus when at least two of the 
following criteria are present: The size of HCC is more than $5 \mathrm{~cm}$, the distance between the portal vein thrombus and the $\mathrm{HCC}$ is less than $2 \mathrm{~cm}$, presence of enhancement within the thrombus itself. Otherwise, the thrombus is considered bland thrombus. According to Sandrasegaran et al. ${ }^{(9)}$, when the formentioned criteria present, diagnosis of malignant thrombosis could be made with a sensitivity of $94-100 \%$ and specificity of $85-90 \%$. They depend on the follow up of the patients to prove the nature of benign thrombi. They considered thrombus that was stable for at least 12 months to be benign. A rapid increase (i.e., within 3 months) in the size of the thrombus to involve the main portal vein and right or left branch during anticoagulation therapy was deemed to indicate malignant thrombus.

\section{DISCUSSION}

Neoplastic portal vein thrombi in patients with HCC gravely affect prognosis and subsequent treatment options. These patients are considered unsuitable for most therapeutic options. Although the reference standard in the diagnosis of the malignant portal vein thrombosis is the pathologic examination; it is an invasive procedure with many drawbacks and therefore imaging plays a major role in differentiation. In our study we found a significant difference between the ADC values of the benign and malignant thrombus $(\mathrm{P}=0.000035)$ and significant statistical difference in the ADC ratio between malignant and bland thrombosis $(\mathrm{P}=$ 0.000755 ) with a cutoff value of $1.25 \mathrm{~mm} 2 / \mathrm{sec}$ to differentiate between neoplastic and bland portal vein thrombi with $85 \%$ sensitivity and $81 \%$ specificity. These findings are supported by the results of previous studies ${ }^{(2,11,12)}$ and ${ }^{(13)}$. To our knowledge, there are no available studies disagree with these results. In our study, we didn't use portal vein caliber as a definite criterion, as its variable. In contrast to Catalano et al. ${ }^{(2)}$ study that considers main portal vein measurement larger than $18 \mathrm{~mm}$ indicating malignancy. Although these criteria had a reasonable sensitivity of $81 \%$ but a specificity of only $48 \%$, many benign acute portal vein thromboses showed venous distention more than $1.8 \mathrm{~cm}$. Also vessel expansion can be observed in patients with portal hypertension without vessel thrombosis. So portal vein caliber cannot be used alone as a good indicator of the nature of thrombus. Also we did not find any statistically significant difference between the $\mathrm{ADC}$ value of the HCC in the neoplastic and bland groups $(\mathrm{P}=0.635525)$ and these finding are similar those of Catalano et al. ${ }^{(2)}$. Our study had some limitations. First, the absence of pathological diagnosis of malignant portal vein thrombus; instead, we used definite accepted imaging criteria as a reference standard that was used in previous studies. As mentioned before, the pathological diagnosis has many limitations. In our study, we tried to avoid averaging the ADC of the thrombus with the surrounding structures by enlarging the images and placing the ROI within the thrombus but we cannot completely exclude that some averaging could have occurred in the smaller thrombi. Another limitation is that the primary hepatic lesion may not always be visible on a nonenhanced study, and some of small welldifferentiated HCC could manifest with diffusion signal and ADC values similar to those of the liver, confounding selection of primary HCC as reference to $\mathrm{ADC}$ ratio. However, in our study we did not find any of such cases. Also, our sample size was small (although the largest to date); a larger study with more statistical power would therefore be helpful to confirm the results of our preliminary investigation.

\section{CONCLUSION}

In conclusion, the diffusion MR imaging can help in the differentiation between malignant and bland portal vein thrombosis in patients with hepatocellular carcinoma using the ADC ratio between the portal vein thrombus and HCC, and therefore affecting the management options.

\section{CONFLICTS OF INTEREST}

There are no conflicts of interest.

\section{REFERENCES}

1- Choi JY, Lee JM and Sirlin CB (2014): CT
and MR imaging diagnosis and staging of
hepatocellular carcinoma: part I.
Development, growth, and spread: key
pathologic and imaging aspects.
Radiology, 272 (3): 635-54.


2- Catalano OA, Choy G, Zhu A et al. (2010): Differentiation of malignant thrombus from bland thrombus of the portal vein in patients with hepatocellular carcinoma: application of diffusion-weighted MR imaging. Radiology, 254 (1): 154-62.

3- Tarantino L, Francica G, Sordelli I et al. (2006): Diagnosis of benign and malignant portal vein thrombosis in cirrhotic patients with hepatocellular carcinoma: color Doppler US, contrastenhanced US, and fine-needle biopsy. Abdom Imaging, 31 (5): 537-44.

4- Shah ZK, McKernan MG, Hahn PF et al. (2007): Enhancing and expansile portal vein thrombosis: value in the diagnosis of hepatocellular carcinoma in patients with multiple hepatic lesions. AJR Am J Roentgenol., 188 (5): 1320-3.

5- Willatt JM, Hussain HK, Adusumilli $S$ et al. (2008): MR Imaging of hepatocellular carcinoma in the cirrhotic liver: challenges and controversies. Radiology, 247 (2): 311-30.

6- Kubo S, Takemura S, Yamamoto $S$ et al. (2007): Risk factors for massive blood loss during liver resection for hepatocellular carcinoma in patients with cirrhosis. Hepatogastroenterology, 54 (75): 830-3.

7- Tublin ME, Dodd GD, Baron RL (1997): Benign and malignant portal vein thrombosis: differentiation by CT characteristics. AJR Am J Roentgenol., 168 (3): 719-23.
8- Peak AS and Sheller A (2007): Risk factors for developing gadolinium-induced nephrogenic systemic fibrosis. Ann Pharmacother., 41 (9): 1481-5.

9- Sandrasegaran K, Tahir B, Nutakki K et al. (2013): Usefulness of conventional MRI sequences and diffusion-weighted imaging in differentiating malignant from benign portal vein thrombus in cirrhotic patients. AJR Am J Roentgenol., 201 (6): 1211-9.

10- Sakata J, Shirai Y, Wakai T et al. (2008): Preoperative predictors of vascular invasion in hepatocellular carcinoma. Eur J Surg Oncol., 34 (8): 900-5.

11- Koh DM and Collins DJ (2007): Diffusion-weighted MRI in the body: applications and challenges in oncology. AJR Am J Roentgenol., 188 (6): 1622-35.

12- Yoshikawa T, Kawamitsu H, Mitchell DG et al. (2006): ADC measurement of abdominal organs and lesions using parallel imaging technique. AJR Am J Roentgenol., 187 (6): 1521-30.

13- Ichikawa T, Haradome $H$, Hachiya $\mathrm{J}$ et al. (1998): Diffusion-weighted MR imaging with a single-shot echoplanar sequence: detection and characterization of focal hepatic lesions. AJR Am J Roentgenology, 170 (2): 397-402. 